Vietnam Journal of Mechanics, VAST, Vol.39, No. 4 (2017), pp. 329 - 338

DOI:10.15625/0866-7136/9693

\title{
NONLINEAR VIBRATION OF FUNCTIONALLY GRADED MATERIAL SANDWICH DOUBLY CURVED SHALLOW SHELLS REINFORCED BY FGM STIFFENERS. PART 2: NUMERICAL RESULTS AND DISCUSSION
}

\author{
Dang Thuy Dong ${ }^{1, *}$, Dao Van Dung ${ }^{2}$ \\ ${ }^{1}$ University of Transport Technology, Hanoi, Vietnam \\ ${ }^{2}$ VNU University of Science, Hanoi, Vietnam \\ *E-mail: dongdt@utt.edu.vn \\ Received April 19, 2017
}

\begin{abstract}
In part 1, the governing nonlinear dynamic equations of FGM sandwich doubly curved shallow shells reinforced by FGM stiffeners on elastic foundation subjected to mechanical and thermal loading are established based on the first order shear deformation theory (FSDT) with von Kármán - type nonlinearity and smeared stiffener technique. In the present part, the fourth-order Runge-Kutta method is applied to investigate influences of models of the shells, FGM stiffeners, thermal environment, elastic foundation, and geometrical parameters on the natural frequencies and dynamic nonlinear responses of stiffened FGM sandwich doubly curved shallow shells.
\end{abstract}

Keywords: Nonlinear vibration, FGM sandwich shallow shell, stiffener.

\section{INTRODUCTION}

Analytical expressions for determining natural frequencies, nonlinear frequencyamplitude relation, and time-deflection curves of stiffened FGM sandwich doubly curved shallow shells are obtained in Part 1 of the paper [1]. In the present part, influences of FGM stiffeners, models of the shells, thermal environment, foundation, and geometrical parameters on dynamic nonlinear responses of sandwich shells are numerically investigated.

Many different methods were applied to investigate nonlinear dynamic response of FGM plates and shells. By using finite element method Parandvar and Farid [2] studied vibration of FGM plate in thermal environment subjected to simultaneously static pressure and harmonic force. Pandey and Pradyumna [3] presented Newmark average acceleration method to consider vibration of FGM sandwich plates and shell panels subjected to rapid heating. Wang and Shen [4] and Bich et al. [5,6] employed Runge-Kutta

(C) 2017 Vietnam Academy of Science and Technology 
method to investigate nonlinear dynamic response of plate and stiffened doubly curved thin shallow shells.

The equations in Part 1 are used to calculate natural frequencies and to plot frequency-amplitude curves and dynamic response curves respectively as follow: Eqs. (26), (28); Eq. (30) and Eqs. (23)-(25) for FGM sandwich shallow shell subjected to mechanical loads and Eqs. (35), (37); Eq. (38) and Eqs. (32)-(34) for FGM sandwich shallow shell subjected to combined mechanical and thermal loads. These equations and equation systems are solved by the fourth-order Runge-Kutta method. Numerical results show effects of FGM stiffeners, models of the sandwich shells, thermal environment, foundation, and geometrical parameters on dynamic nonlinear responses of sandwich shells. In addition, the comparison results between the classical shell theory (CST) and the first-order shear deformation theory (FSDT) are considered. It is noted that for $R_{x}=R_{y}$, it corresponds to the spherical panel (S-panel); for $R_{x} \rightarrow \infty$, it corresponds to the cylindrical panel (C-panel); for $R_{x}=-R_{y}$, it corresponds to the hyperbolic paraboloidal panel (Hpanel) and for $R_{x} \rightarrow \infty, R_{y} \rightarrow \infty$ it corresponds to the plate.

\section{NUMERICAL RESULTS AND DISCUSSIONS}

\subsection{Comparison studies}

In order to verify the analytical formulation presented in the previous part, firstly, the natural frequencies of un-stiffened FGM square sandwich plate with model 1B are compared with those of [7] based on the sinusoidal shear deformation plate theories. The input data is $E_{m}=70 \mathrm{GPa}, \rho_{m}=2707 \mathrm{~kg} / \mathrm{m}^{3} ; E_{c}=380 \mathrm{GPa}, \rho_{c}=3800 \mathrm{~kg} / \mathrm{m}^{3} ; v=0.3$, $K_{s}=5 / 6, h_{t}=h_{b}=h / 3, a / h=10$. From the result listed in Tab. 1 , it is observed that the compared results are in good agreement.

Table 1. Comparison of fundamental frequency $\bar{\omega}=\omega a^{2} / h \sqrt{\rho_{0} / E_{0}}\left(E_{0}=1 \mathrm{Gpa}\right.$ and $\left.\rho_{0}=1 \mathrm{~kg} / \mathrm{m}^{3}\right)$ of un-stiffened FGM square sandwich plate $((m, n)=(1,1))$

\begin{tabular}{|c|c|c|c|c|c|}
\hline Theory & $k=0$ & $k=0.5$ & $k=1$ & $k=5$ & $k=10$ \\
\hline CLPT [7] & 1.87359 & 1.54903 & 1.37521 & 1.05565 & 1.00524 \\
\hline FSDPT [7] & 1.82442 & 1.51695 & 1.35072 & 1.04183 & 0.99256 \\
\hline TSDPT [7] & 1.82445 & 1.51922 & 1.35333 & 1.04466 & 0.99551 \\
\hline SSDPT [7] & 1.82452 & 1.51927 & 1.35339 & 1.04481 & 0.99519 \\
\hline FSDT[Present] & 1.82617 & 1.51713 & 1.35044 & 1.04138 & 0.99218 \\
\hline
\end{tabular}

CLPT: the classical plate theory, FSDPT: the first-order shear deformation plate theory, TSDPT: the third-order shear deformation plate theory, SSDPT: the sinusoidal shear deformation theory.

Next, we have a comparison between the natural frequency of the FGM spherical panel reinforced (model 2A with $h_{t}=h_{b}=0 \mathrm{~m}$ ) by homogenous stiffeners without elastic foundation obtained in [1], and the results were calculated by Eq. (32) of [6] based on the CST. The shell is considered with the following characteristics: $a=b=1 \mathrm{~m}$, $R_{x}=R_{y}=5 \mathrm{~m}, h_{x}=h_{y}=0.01 \mathrm{~m}, v=0.3, d_{x}=d_{y}=0.003 \mathrm{~m}, s_{x}=s_{y}=0.1 \mathrm{~m}, E_{m}=70$ GPa, $\rho_{m}=2707 \mathrm{~kg} / \mathrm{m}^{3}, E_{c}=380 \mathrm{GPa}, \rho_{c}=3800 \mathrm{~kg} / \mathrm{m}^{3}$ and $(m, n)=(1,1)$. 
Table 2. Comparison of fundamental frequency of FGM spherical panel reinforced by homogenous stiffeners

\begin{tabular}{|c|c|c|c|c|}
\hline$a / h$ & $k$ & CPT [6] & FSDT [Present] & Difference $(\%)$ \\
\hline \multirow{4}{*}{200} & 0.2 & 1817.10 & 1814.37 & 0.15 \\
\cline { 2 - 5 } & 1 & 1575.89 & 1573.70 & 0.14 \\
\cline { 2 - 5 } & 5 & 1225.26 & 1222.93 & 0.19 \\
\hline \multirow{4}{*}{100} & 0.2 & 1926.99 & 1916.17 & 0.56 \\
\cline { 2 - 5 } & 1 & 1669.87 & 1661.47 & 0.50 \\
\cline { 2 - 5 } & 5 & 1309.15 & 1301.09 & 0.62 \\
\hline \multirow{4}{*}{50} & 0.2 & 2174.77 & 2134.71 & 1.84 \\
\cline { 2 - 5 } & 1 & 1868.41 & 1837.10 & 1.68 \\
\cline { 2 - 5 } & 5 & 1493.08 & 1463.84 & 1.96 \\
\hline \multirow{4}{*}{20} & 0.2 & 3350.90 & 3176.43 & 5.21 \\
\cline { 2 - 5 } & 1 & 2809.96 & 2670.88 & 4.95 \\
\cline { 2 - 5 } & 5 & 2345.56 & 2219.95 & 5.36 \\
\hline \multirow{3}{*}{10} & 0.2 & 5854.05 & 5397.34 & 7.80 \\
\cline { 2 - 5 } & 1 & 4845.91 & 4483.50 & 7.48 \\
\cline { 2 - 5 } & 5 & 4134.36 & 3804.81 & 7.97 \\
\hline \multirow{3}{*}{5} & 0.2 & 11243.72 & 9855.91 & 12.34 \\
\cline { 2 - 5 } & 1 & 9263.49 & 8200.99 & 11.47 \\
\cline { 2 - 5 } & 5 & 7965.25 & 6943.13 & 12.83 \\
\hline
\end{tabular}

As seen in Tab. 2, for thin shell $(a / h \geq 50)$, we obtain a good agreement. However, for thicker shells $(5 \leq a / h<50)$ the difference between two theories is considerable. Therefore, the FSDT may be necessary to obtain reasonably accurate solutions for a thicker shell.

\subsection{Numerical results for FGM sandwich shallow shells}

In this section, we consider perfect and imperfect FGM sandwich doubly curved shallow shells reinforced by FGM stiffeners on elastic foundation. The geometric and material properties of shell and stiffeners are $a=b=1 \mathrm{~m}, a / h=30, h_{t}=h_{b}, R_{x}=$ $R_{y}=5 \mathrm{~m}, k_{t}=k_{b}=k ; h_{x}=h_{y}, d_{x}=d_{y}, s_{x}=s_{y}=0.1 \mathrm{~m}, k_{2}=k_{3}=1 / k$ and $K_{1}=5 \times 10^{7} \mathrm{~N} / \mathrm{m}^{3}, K_{2}=2 \times 10^{5} \mathrm{~N} / \mathrm{m}$ (unless otherwise). The shear correction coefficient is chosen to be $K_{s}=5 / 6$. The shells are composed from mixture of silicon nitride $\left(\mathrm{Si}_{3} \mathrm{~N}_{4}\right)$ and stainless steel (SUS304) according to the sigmoid or power distribution laws. In the FGM layers, effective value of material properties Pr, such as Young's modulus $E$, thermal expansion coefficient $\alpha$ and the mass density $\rho$ can be expressed 
as [8] $\operatorname{Pr}=P_{0}\left(P_{-1} T^{-1}+1+P_{1} T+P_{2} T^{2}+P_{3} T^{3}\right)$, where $T=T_{0}+\Delta T$ and $T_{0}=300 \mathrm{~K}$ (room temperature), $P_{0}, P_{-1}, P_{1}, P_{2}, P_{3}$ are the coefficients in which typical values (including Young's modulus $E$, thermal expansion coefficient $\alpha$ and mass density $\rho$ ) are adopted as in Reddy and Chin [9]. Poisson's ratio is assumed to be a constant and $v=0.29$.

\subsubsection{Natural frequencies}

A comparison between the natural frequencies of the shell calculated by the approximate Eq. (28) (assuming that $\ddot{\Phi}_{x}, \ddot{\Phi}_{y}$ are ignored) and by the full Eq. (26) is shown in Tab. 3. It can see that there is a small difference between the natural frequencies calculated by these equations. Therefore, in order to simplify, $\ddot{\Phi}_{x}$ and $\ddot{\Phi}_{y}$ will be ignored in the remainder of numerical examples.

Table 3. Comparisons of the natural frequencies of S-panel calculated by Eq. (26) and Eq. (28) $\left(h_{t} / h=0.3, k_{t}=1, h_{x}=0.03 \mathrm{~m}, d_{x}=0.006 \mathrm{~m}\right)$

\begin{tabular}{|c|c|c|c|}
\hline Modeling & Using Eqs. (26) & Using Eqs. (28) & Difference (\%) \\
\hline 1A & 1783.60 & 1780.89 & 0.15 \\
\hline 1B & 2251.11 & 2246.45 & 0.20 \\
\hline 2A & 1987.56 & 1985.12 & 0.12 \\
\hline 2B & 1957.51 & 1953.59 & 0.2 \\
\hline
\end{tabular}

Tab. 4 shows that the natural frequency of the FGM sandwich shell with model 2A is larger than natural frequency of normal FGM shell with the same thickness. Besides, the elastic foundations also have influences on the natural frequency of the shell. Specifically, natural frequency of the shell on Pasternak elastic foundation is the largest and that of the shell without elastic foundation is the smallest. From the results in this table we can also see that the temperature considerably decrease natural frequency of the shell.

Table 4. Comparisons of natural frequencies of FGM shells and FGM sandwich shells with model $2 \mathrm{~A}\left(h_{t} / h=1 / 3, k_{t}=1, h_{x}=0.02 \mathrm{~m}, d_{x}=0.006 \mathrm{~m}\right)$

\begin{tabular}{|c|c|c|c|c|c|c|}
\hline \multirow{2}{*}{$\Delta T(K)$} & \multicolumn{2}{|c|}{ Pasternak foundation } & \multicolumn{2}{|c|}{ Winkler foundation } & Without foundation \\
\cline { 2 - 7 } & FGM & $\begin{array}{c}\text { FGM } \\
\text { sandwich }\end{array}$ & FGM & $\begin{array}{c}\text { FGM } \\
\text { sandwich }\end{array}$ & FGM & $\begin{array}{c}\text { FGM } \\
\text { sandwich }\end{array}$ \\
\hline$\Delta T=0$ & 2515.73 & 2529.84 & 2511.55 & 2525.68 & 2457.98 & 2472.44 \\
\hline$\Delta T=300$ & 2438.35 & 2452.50 & 2434.03 & 2448.21 & 2378.73 & 2393.24 \\
\hline$\Delta T=600$ & 2319.01 & 2334.68 & 2314.48 & 2330.17 & 2256.24 & 2272.35 \\
\hline
\end{tabular}

Results obtained from Tab. 5 point out that the shell reinforced by FGM stiffener has natural frequency larger than the shell reinforced by homogeneous stiffeners. The stiffener arrangement also influences to the natural frequency of the shell. With S-panel, both arrangements on $x$ direction and $y$ direction give the same natural frequency. However, using orthogonal stiffeners will give larger natural frequency. These predictions have important significances for stiffened shell manufacturing. Moreover, we can see 
that the volume fraction index $k$ significantly influences to natural frequency of the shell. Specifically, with model 1B, the natural frequency of the shell remarkably decreases as $k$ increases.

Table 5. Effect of FGM stiffeners on natural frequencies of S-Panel with model 1B $\left(h=a / 40, h_{t} / h=0.5, h_{x}=0.025 \mathrm{~m}, d_{x}=0.006 \mathrm{~m}, \Delta T=200 \mathrm{~K}\right)$

\begin{tabular}{|c|c|c|c|}
\hline$k$ & Type of stiffeners & FGM stiffeners & Homogeneous stiffeners \\
\hline \multirow{3}{*}{$k=0.2$} & Transversal stiffeners & 1484.03 & 1515.18 \\
\cline { 2 - 4 } & Longitudinal stiffeners & 1484.03 & 1515.18 \\
\cline { 2 - 4 } & Orthogonal stiffeners & 1487.01 & 1519.02 \\
\hline \multirow{3}{*}{$k=1$} & Transversal stiffeners & 1076.31 & 1020.87 \\
\cline { 2 - 4 } & Longitudinal stiffeners & 1076.31 & 1020.87 \\
\cline { 2 - 4 } & Orthogonal stiffeners & 1082.22 & 1024.25 \\
\hline \multirow{3}{*}{$k=5$} & Transversal stiffeners & 829.49 & 761.59 \\
\cline { 2 - 4 } & Longitudinal stiffeners & 829.49 & 761.59 \\
\cline { 2 - 4 } & Orthogonal stiffeners & 837.72 & 765.57 \\
\hline
\end{tabular}

Table 6. Comparisons of natural frequencies for four models of S-panel $\left(h_{x}=0.02 \mathrm{~m}, d_{x}=0.005 \mathrm{~m}\right)$

\begin{tabular}{|c|c|c|c|c|c|c|}
\hline \multirow{2}{*}{ Modeling } & \multicolumn{2}{|c|}{$h_{t} / h=0.2$} & \multicolumn{2}{c|}{$h_{t} / h=0.3$} & \multicolumn{2}{c|}{$h_{t} / h=0.5$} \\
\cline { 2 - 7 } & T-ID & T-D & T-ID & T-D & T-ID & T-D \\
\hline 1A & 1676.21 & 1572.60 & 1786.11 & 1677.19 & 2026.56 & 1904.15 \\
\hline 1B & 2029.02 & 1822.00 & 1813.68 & 1615.07 & 1493.95 & 1307.03 \\
\hline 2A & 1866.59 & 1725.76 & 1878.66 & 1739.35 & 1897.64 & 1761.09 \\
\hline 2B & 1699.94 & 1550.22 & 1700.25 & 1551.63 & 1710.90 & 1565.09 \\
\hline
\end{tabular}

Influence of ratio $h_{t} / h$ on the natural frequency of the shell in cases of temperature dependent (TD) and temperature independent material properties (T-ID) is investigated in Tab. 6 . The results show that when ratio $h_{t} / h$ raises, natural frequencies of model $1 \mathrm{~A}$, $2 \mathrm{~A}$, and $2 \mathrm{~B}$ increase while natural frequency of model $1 \mathrm{~B}$ decreases. In the other hand, shell's natural frequency in T-ID case is larger than in TD case.

\subsubsection{Frequency-amplitude curves}

In this section, we consider a stiffened FGM sandwich shallow shell with $h=a / 40$, $h_{t} / h=0.5, h_{x}=0.02 \mathrm{~m}, d_{x}=0.005 \mathrm{~m}$.

The frequency-amplitude curves of nonlinear free vibration and nonlinear forced vibration of the kinds of doubly curved shallow shells is shown in Fig. 1. These frequencyamplitude curves of three types of shell are notably different. In which, extreme point of the frequency-amplitude curve is the smallest with S-panel and the largest with H-panel. 


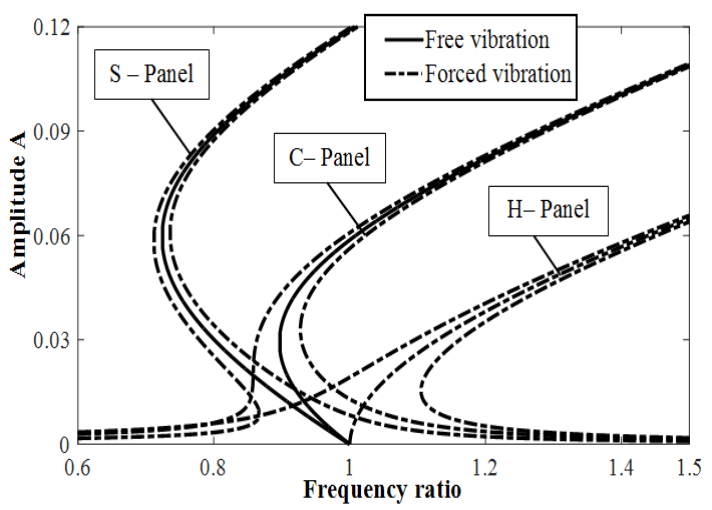

Fig. 1. The frequency-amplitude curves of the kinds of the shell with model $1 \mathrm{~A}$ $\left(Q=3 \times 10^{5} \mathrm{~N} / \mathrm{m}^{2}\right)$

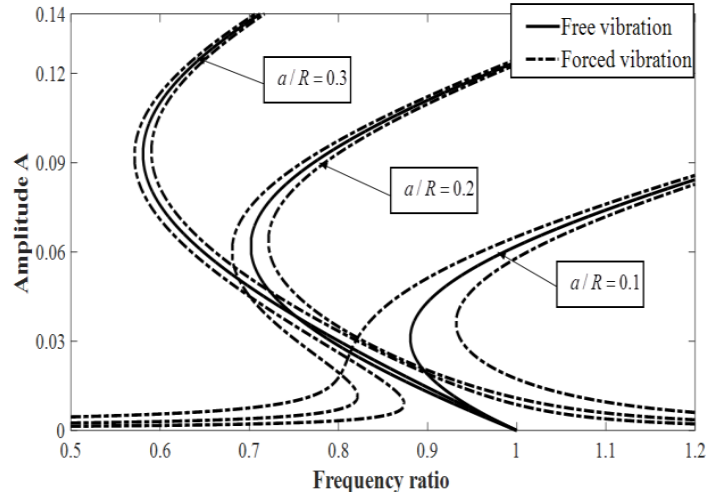

Fig. 2. Effect of $a / R$ ratio on the frequencyamplitude curve of S-panel with model $1 \mathrm{~A}$ $\left(Q=5 \times 10^{5} \mathrm{~N} / \mathrm{m}^{2}\right)$

Fig. 2 investigates the effect of $a / R$ ratio on the frequency-amplitude curve of the shallow spherical panel. As can be seen, when $a / R$ ratio decreases or the curvature of the shell decreases, the extreme point of the frequency-amplitude curve increase.

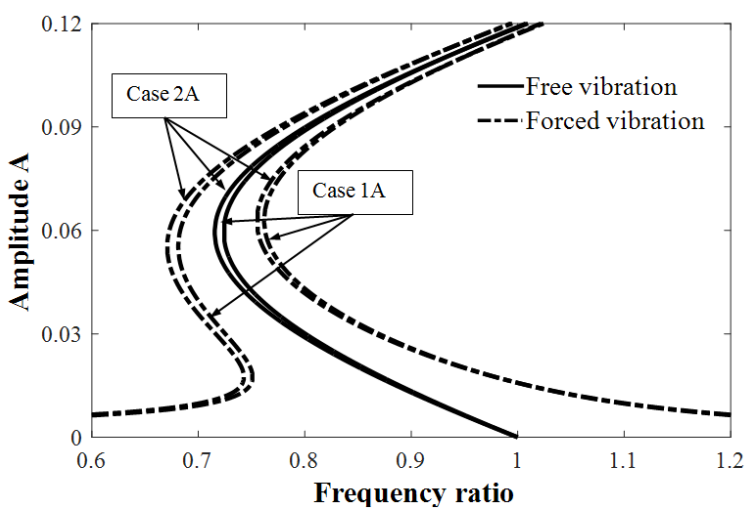

Fig. 3. The frequency-amplitude curves of the S-panel with models $1 \mathrm{~A}$ and $2 \mathrm{~A}\left(Q=10^{6} \mathrm{~N} / \mathrm{m}^{2}\right)$

Fig. 3 compares frequency-amplitude curves of S-Panel with model 1A (symmetric sigmoid law) and S-Panel with case 2A (symmetric power law). The extreme point of the frequency-amplitude curve in case of $1 \mathrm{~A}$ is larger than in case of $2 \mathrm{~A}$. However, the deviation is not large.

\subsubsection{Nonlinear dynamic response curves}

To investigate the dynamic response curves, the geometric and material properties of FGM sandwich shallow shells and stiffeners are chosen as: $a=b=1 \mathrm{~m}, h=a / 30$, $h_{t} / h=h_{b} / h, k_{t}=1, h_{x}=0.03 \mathrm{~m}, d_{x}=0.006 \mathrm{~m}, s_{x}=0.1 \mathrm{~m}$.

Fig. 4 and Fig. 5 describe the influence of FGM stiffeners on nonlinear dynamic response curves in case of the shell under mechanical load and thermo-mechanical load. Obviously, in both cases, shell reinforced by FGM stiffener has deflection amplitude smaller than un-stiffened shell. Hence, besides increasing natural frequency of shells, 
FGM stiffeners also reduce the deflection of the shell when they are under external pressure and thermal loads.

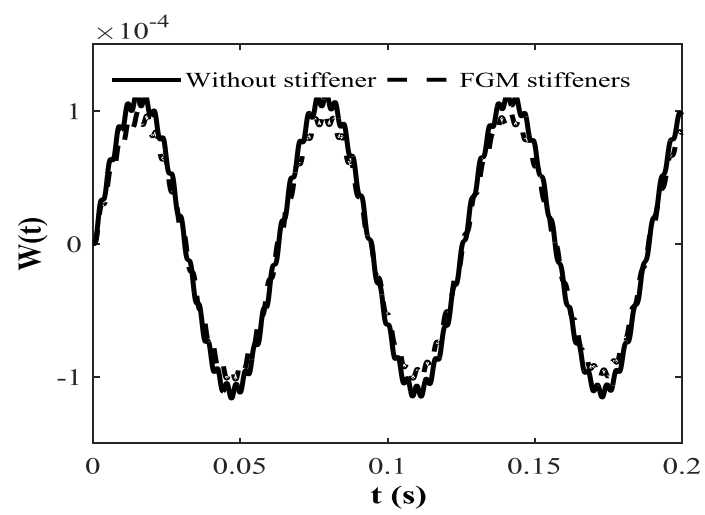

Fig. 4. Effect of FGM stiffener on dynamic response curves of S-panel under external pressure $\left(q=5 \times 10^{4} \sin (100 t), h_{t} / h=0.5 \varepsilon=2\right.$,

$$
\left.\xi=10^{-4}\right)
$$

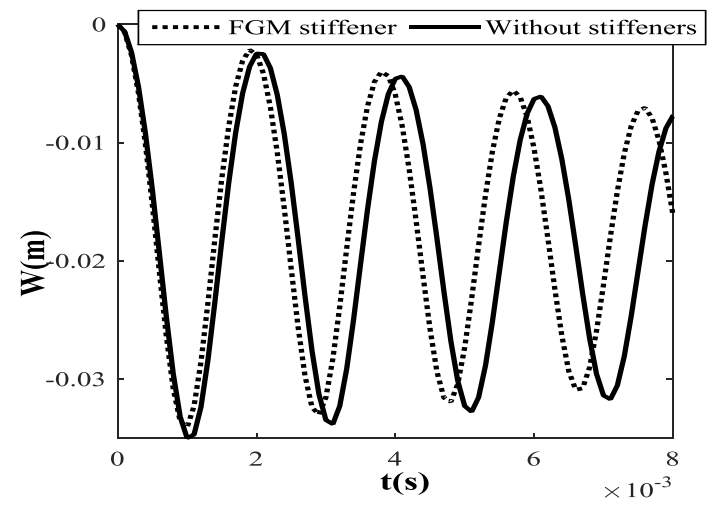

Fig. 5. Effect of FGM stiffener on dynamic response curves of S-panel under external pressure and temperature environment $\left(\Delta T=300 \mathrm{~K}, q=5 \times 10^{4} \sin (100 t)\right.$,

$$
\left.h_{t} / h=0.5 \varepsilon=50, \xi=10^{-4}\right)
$$

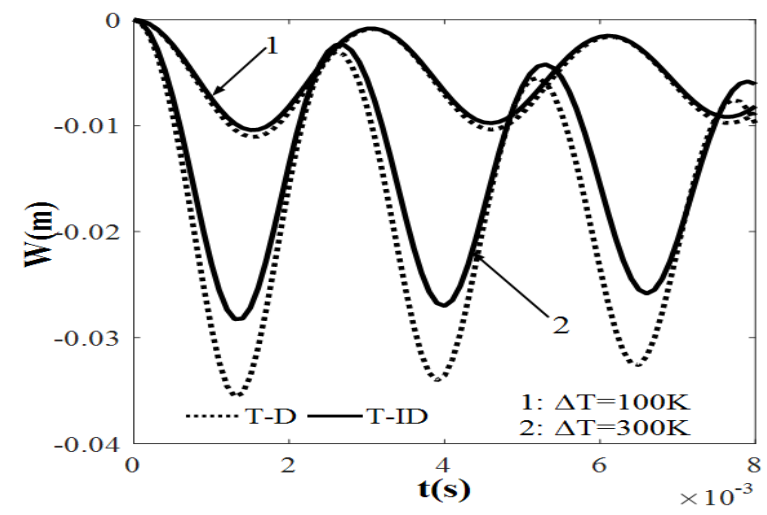

Fig. 6. Effect of temperature on dynamic response curves of S-panel $\left(h_{t} / h=0.1, q=5 \times 10^{4} \sin (100 t), \varepsilon=50, \xi=10^{-4}, \Delta T=300 \mathrm{~K}\right)$

Effect of the temperature on nonlinear dynamic response curve of S-Panel is investigated in Fig. 6. Observably, when temperature increases, the amplitude of timedeflection curve increases. At the same time, in TD case, this amplitude is larger than in T-ID case. Besides, when the shell under thermal load, the deflection amplitude only has negative zone without positive zone as in case of shell under mechanical load.

Fig. 7 and Fig. 8 compare nonlinear dynamic response of model 1A and model $2 \mathrm{~A}$ in case of shell only under external pressure and in case of shell under both external pressure and thermal environment. If shell is only subjected to external pressure (FM boundary condition), the deflection amplitude of model 1A is larger than of model 2A. 


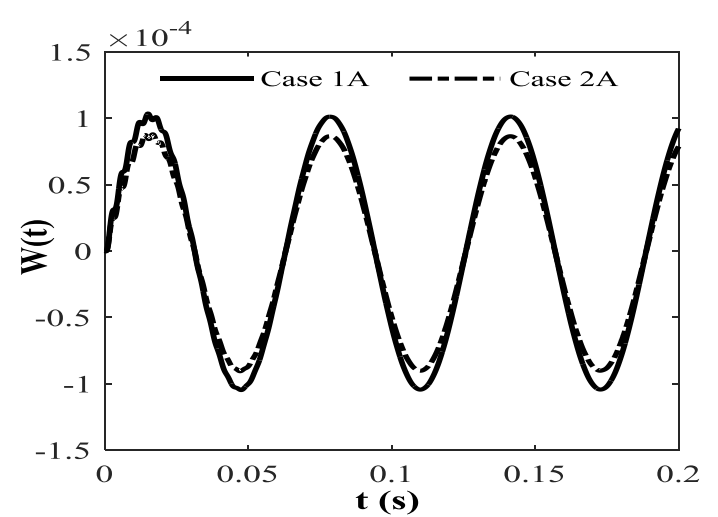

Fig. 7. Dynamic response curves of the S-panel with two models $1 \mathrm{~A}$ and $2 \mathrm{~A}$ in case only under external pressure $\left(h_{t} / h=0.5, q=5 \times\right.$ $\left.10^{4} \sin (100 t), \varepsilon=50, \xi=10^{-4}\right)$

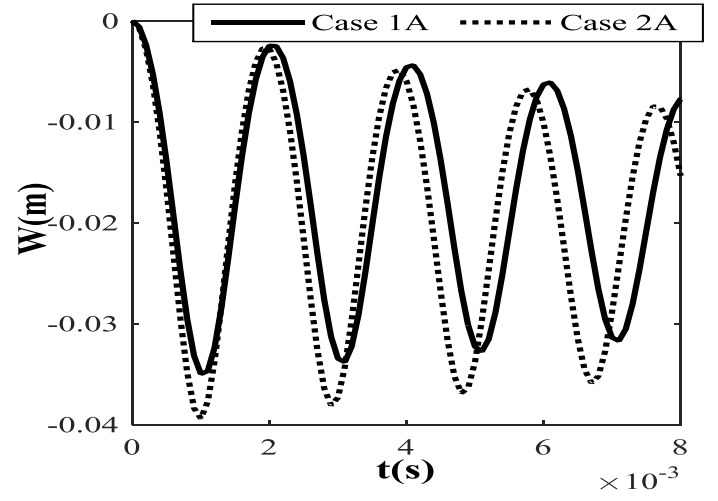

Fig. 8. Dynamic response curves of the S-panel with two models $1 \mathrm{~A}$ and $2 \mathrm{~A}$ in case under external pressure and temperature environment $\left(h_{t} / h=0.5, q=5 \times 10^{4} \sin (100 t), \varepsilon=50\right.$, $\left.\xi=10^{-4}, \Delta T=300 \mathrm{~K}\right)$

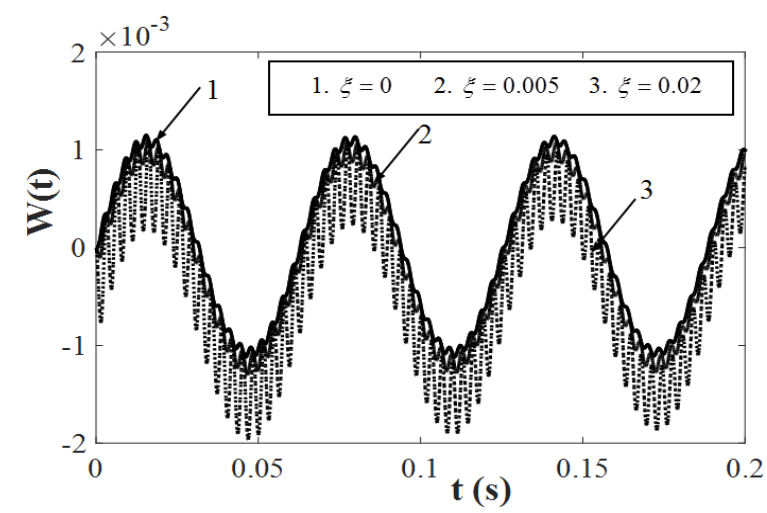

Fig. 9. Effect of the initial imperfection size on dynamic response curves of S-panel under external pressure $\left(h_{t} / h=0.1, q=10^{6} \sin (100 t), \varepsilon=2\right)$

However, with IM boundary condition and with effect of temperature, the reversed trend happens.

Fig. 9 shows the effect of the initial imperfection size on dynamic response curve of the S-panel (model 1A). With positive deflection zone, the imperfection shell's deflection amplitude is smaller than perfect shell's. However, in the negative zone, the perfect shell's deflection amplitude is smaller. In addition, it can be seen that the dynamic response curve is very sensitive to the initial imperfection size.

The time-deflection curves of S-panel when the excitation frequency approaches the shell's natural frequency (as in Tab. 3) is shown in Fig. 10. A similar phenomenon as harmonic beat phenomenon can be observed, in which, the amplitude of the harmonic beat quickly raises when the excitation frequency is near the natural frequency. Besides, under the influence of the damping, the harmonic beat phenomenon does not appear clearly after numbers of vibration periods as in Fig. 11. 


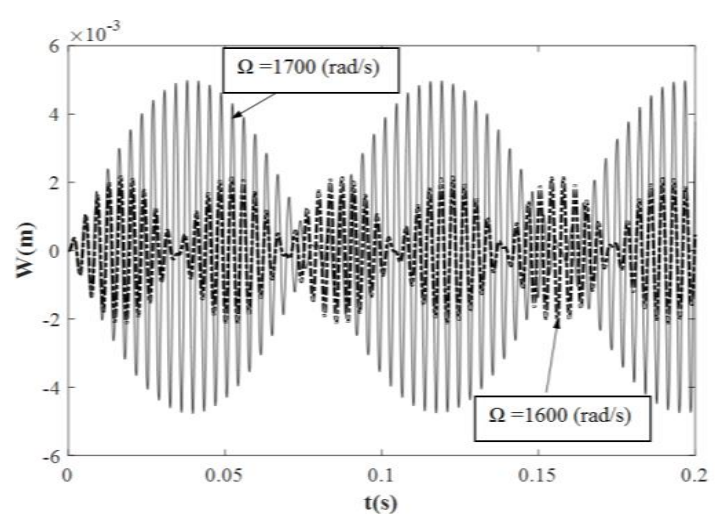

Fig. 10. Effect of excitation frequency on harmonic beat phenomenon of S-panel $(\varepsilon=0$,

$$
\left.Q=10^{5} \mathrm{~N} / \mathrm{m}^{2}, \xi=10^{-5}, h_{t} / h=0.3\right)
$$

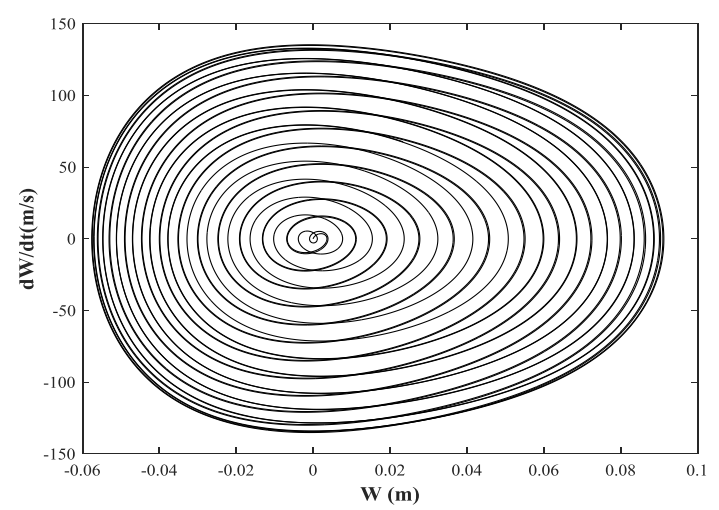

Fig. 12. Deflection-velocity curve of S-panel with $q=10^{6} \sin (1750 t), \xi=10^{-4}, h_{t} / h=0.3$

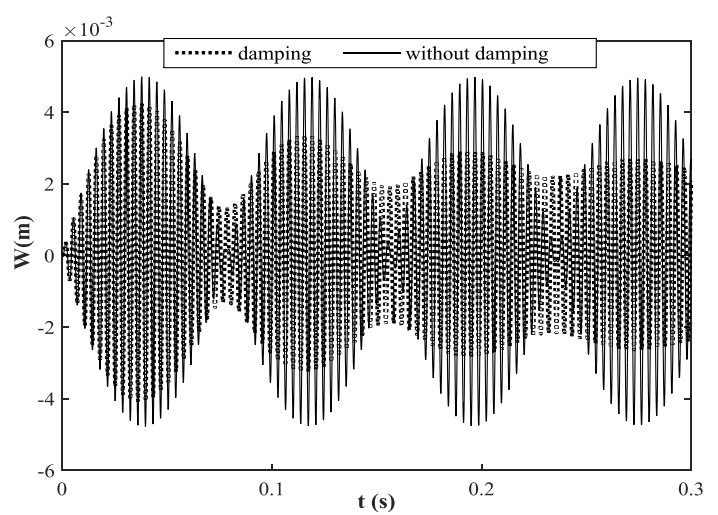

Fig. 11. Effect of the damping on harmonic beat phenomenon of S-panel $\left(\varepsilon=9, Q=10^{5}\right.$ $\left.\mathrm{N} / \mathrm{m}^{2}, \xi=10^{-5}, h_{t} / h=0.3\right)$

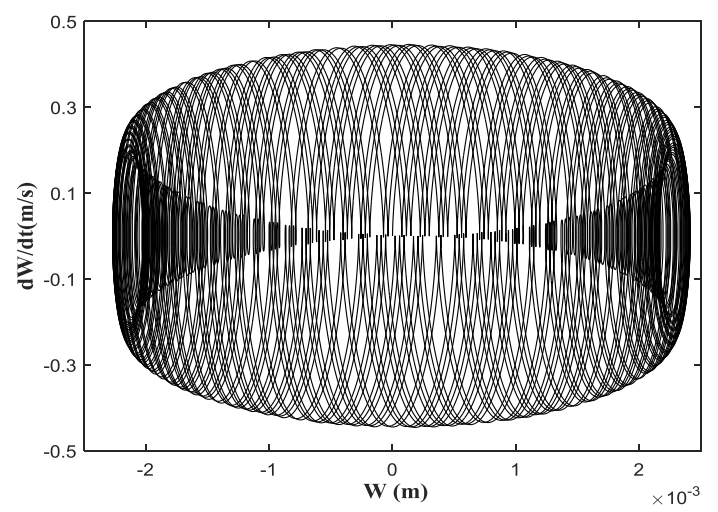

Fig. 13. Deflection-velocity curve of S-panel with $q=10^{6} \sin (100 t), \xi=10^{-4}, h_{t} / h=0.3$

Fig. 12 and Fig. 13 investigate the deflection-velocity curve of S-panel under external pressure. When the excitation frequency is near natural frequency, this curve has a shape of closed spiral as shown in Fig. 12. When the excitation frequency is far from the natural frequency, the curve becomes more turbulent and contains many cross lines as shown in Fig. 13.

\section{CONCLUSION}

By using the analytical expressions and the governing equations in Part 1 and applying Runge-Kutta method, natural frequencies, nonlinear frequency-amplitude relation, and nonlinear dynamic response curves of sandwich shallow shells are investigated in this Part. From the present results, some conclusion can be deduced as follow:

i). Structural models of sandwich shallow shell, FGM layer thickness, kinds of the shell, volume fraction index, elastic foundation, and damping have significant influence 
on the natural frequencies, the nonlinear frequency-amplitude relation, and the nonlinear dynamic response curve.

ii). For model 1B, shell reinforced by FGM stiffeners has natural frequency larger than the shell reinforced by homogeneous stiffeners.

iii). For model 2A, the natural frequency of the FGM sandwich shell is larger than natural frequency of normal FGM shell with the same thickness.

iv). Temperature reduces the natural frequency and increases the deflection amplitude of the dynamic response curve of the sandwich shell. Besides, the shells have smaller natural frequency and larger deflection amplitude when the temperaturedependent material properties are taken into account.

v). The sandwich shell with model of symmetric sigmoid law (Case 1A) has a larger natural frequency and smaller deflection amplitude than model of symmetric power law (Case 2A). The extreme point of the frequency-amplitude curve in case of symmetric sigmoid law is also larger than in case of symmetric power law.

\section{ACKNOWLEDGMENT}

This research is funded by Vietnam National Foundation for Science and Technology Development (NAFOSTED) under grant number 107.02-2015.11.

\section{REFERENCES}

[1] D. T. Dong and D. V. Dung. Nonlinear vibration of functionally graded material sandwich doubly curved shallow shells reinforced by FGM stiffeners. Part 1: Governing equations. Vietnam Journal of Mechanics, 39, (3), (2017), pp. 245-257. doi:10.15625/0866-7136/9692.

[2] H. Parandvar and M. Farid. Large amplitude vibration of FGM plates in thermal environment subjected to simultaneously static pressure and harmonic force using multimodal FEM. Composite Structures, 141, (2016), pp. 163-171. doi:10.1016/j.compstruct.2016.01.044.

[3] S. Pandey and S. Pradyumna. A finite element formulation for thermally induced vibrations of functionally graded material sandwich plates and shell panels. Composite Structures, 160, (2017), pp. 877-886. doi:10.1016/j.compstruct.2016.10.040.

[4] Z. X. Wang and H. S. Shen. Nonlinear dynamic response of sandwich plates with FGM face sheets resting on elastic foundations in thermal environments. Ocean Engineering, 57, (2013), pp. 99-110. doi:10.1016/j.oceaneng.2012.09.004.

[5] D. H. Bich, D. V. Dung, and V. H. Nam. Nonlinear dynamical analysis of eccentrically stiffened functionally graded cylindrical panels. Composite Structures, 94, (8), (2012), pp. 24652473. doi:10.1016/j.compstruct.2012.03.012.

[6] D. H. Bich, D. V. Dung, and V. H. Nam. Nonlinear dynamic analysis of eccentrically stiffened imperfect functionally graded doubly curved thin shallow shells. Composite Structures, 96, (2013), pp. 384-395. doi:10.1016/j.compstruct.2012.10.009.

[7] A. M. Zenkour. A comprehensive analysis of functionally graded sandwich plates: Part 2 - Buckling and free vibration. International Journal of Solids and Structures, 42, (18), (2005), pp. 5243-5258. doi:10.1016/j.ijsolstr.2005.02.016.

[8] Y. S. Touloukian. Thermophysical properties of high temperature solid materials. New York, NY: MacMillan, (1967).

[9] J. N. Reddy and C. D. Chin. Thermomechanical analysis of functionally graded cylinders and plates. Journal of Thermal Stresses, 21, (6), (1998), pp. 593-626. doi:10.1080/01495739808956165. 